\title{
Right ventricular dysfunction during coronary artery occlusion: pressure-volume analysis using conductance catheters during coronary angioplasty
}

Andrew Bishop, Paul White, Peter Groves, Rajiv Chaturvedi, Carl Brookes, Andrew Redington, Paul Oldershaw

\begin{abstract}
Objective-To study the effects of coronary artery occlusion on the pressure-volume relations of the right ventricle.

Design-Right ventricular pressurevolume cycles were studied using conductance catheters and micromanometers in 19 subjects undergoing coronary angioplasty in a tertiary referral cardiac centre.

Results-Catheter occlusions of either the left anterior descending coronary artery or the right coronary artery were associated with a decline in stroke work (mean change (SD): left $-13.3(15.8) \%, p=0.008$; right $-13.5(16.5) \%, p=0.04)$. Two patterns of change were evident: an upward shift usually associated with occlusion in the left coronary artery, and a rightward shift in the right coronary artery. In the former there was an increase in maximum ventricular volume (mean change: $3.0(2.7) \%, p=0.004)$ and in minimum ventricular volume (mean change: $2.3(2.7) \%, p=0.01)$ and $a$ fall in peak pressure (mean change: $-4.8(5.1) \%$, $\mathbf{p}=\mathbf{0 . 0 4})$. In the latter there was an increase in peak pressure (mean change 9.9(16.3)\%, $p=0.04)$ and an increase in minimum ventricular volume (mean change $3.7(5.0) \%, p=0.02)$ leading to a fall in stroke volume (mean change $-13.3(15.8) \%, p=0.008)$.

Conclusions-Occlusion of the left anterior descending coronary artery or the right coronary artery is associated with a decline in right ventricular work. However, different patterns of change in indices of preload and afterload lead to different effects on overall right ventricular pump function.

(Heart 1997;78:480-487)
\end{abstract}

Royal Brompton Hospital, London, UK

Correspondence to: Dr Bishop, Cardiac Department, St Thomas' Hospital, Lambeth Palace Road, London SE1 7EH, UK.

Accepted for publication 22 July 1997
Interest in right ventricular function as an independent factor in the pathophysiology of heart disease is increasing. The initial success of right heart bypass for tricuspid atresia ${ }^{1}$ suggested that the right ventricle was "dispensable". ${ }^{2}$ However, there is now convincing evidence that in patients with coronary artery disease right ventricular dysfunction is of functional ${ }^{3}$ and prognostic ${ }^{4}$ significance. This is most striking in the context of acute inferior myocardial infarction, where direct right ventricular involvement increases the early mortality from $6 \%$ to $31 \%$ in groups matched for the degree of coexisting left ventricular dysfunction. ${ }^{5}$

There is no agreement as to whether right ventricular dysfunction arises primarily from the adverse loading that accompanies an increase in the left ventricular diastolic pressure in patients with ischaemic heart disease, or from intrinsic changes in the active and passive properties of the right ventricular myocardium when it is ischaemic. In animal models an inverse relation between stroke volume and peak ventricular pressure shows the normal right ventricle to be twice as sensitive to an increased afterload as the left. ${ }^{6}$ In humans, results from apparently similar studies investigating the effect of right ventricular ischaemia are in direct conflict, suggesting that right coronary artery disease is a significant ${ }^{7}$ or insignificant ${ }^{8}$ determinant of the right ventricular ejection fraction during exercise.

Pressure-volume analysis, the derivation of the relation between simultaneous ventricular volume and pressure during the cardiac cycle, is a powerful method of distinguishing changes in ventricular loading from changes in the active and passive properties of the myocardium. Left ventricular pressure-volume studies are now routinely reported in animal and human subjects using a volume signal from a conductance catheter. This technique has the advantage of continuous and instantaneous display and acquisition of simultaneous pressure and volume to create real time pressurevolume cycles. Since conductance derived volumes are not derived from an image, and can be monitored during periods of change, they are potentially useful in assessing the response of the right ventricle to ischaemia.

The use of conductance catheters has recently been described in right ventricular models, ${ }^{9}$ and in animal ${ }^{1011}$ and human ${ }^{12}$ subjects. In this study we describe the use of conductance catheters and catheter mounted micromanometers to investigate the pressurevolume relations of the human right ventricle during coronary angioplasty. The ischaemia that occurs during coronary occlusion is interpreted as a model of the prolonged ischaemia 
Table 1 Percentage change in indices derived from right ventricular pressure-volume cycles after 60 seconds of coronary occlusion in patients undergoing coronary angioplasty

\begin{tabular}{|c|c|c|c|c|c|c|c|c|c|}
\hline Patient & Coronary disease & Dominance & $\max P$ & $\max V$ & $\min V$ & $s v$ & $s w$ & $d P / d t m a x$ & $\begin{array}{l}\text { Cycle } \\
\text { efficiency }\end{array}$ \\
\hline 1 & Mid LAD & RCA & 18.6 & 0.7 & 1.8 & -5.6 & 1.7 & 8.7 & -13.0 \\
\hline 2 & Proximal LAD & RCA & 5.6 & 0.1 & 0.2 & -1.6 & -3.0 & 0.0 & 5.8 \\
\hline 3 & Mid LAD & RCA & 0.2 & 5.6 & 8.3 & -8.8 & -15.7 & 8.5 & -17.9 \\
\hline 4 & Mid LAD & RCA & 42.3 & 0.8 & 10.6 & -41.5 & -55.6 & 21.1 & -78.3 \\
\hline 5 & Mid LAD & RCA & -9.5 & 3.9 & 11.7 & -41.4 & -50.4 & 21.1 & -12.2 \\
\hline 6 & Mid LAD & RCA & 4.0 & -3.6 & -4.1 & -0.5 & -23.8 & 32.6 & -14.9 \\
\hline 7 & Proximal LAD & RCA & -4.5 & 1.0 & 3.5 & -14.6 & -44.2 & -13.8 & -62.2 \\
\hline 8 & Proximal D1 & RCA & 9.7 & 0.7 & 2.7 & -12.9 & 1.9 & 9.2 & 13.1 \\
\hline 9 & Mid LAD & RCA & 1.1 & -1.5 & -0.2 & -9.6 & -70.0 & -15.2 & 1.5 \\
\hline \multirow[t]{2}{*}{10} & Mid LAD, distal LAD & RCA & 31.6 & 2.4 & 2.2 & 3.4 & 50.2 & 24.0 & 2.0 \\
\hline & Proximal OM1 & & -1.0 & 3.8 & 0.2 & 20.8 & -4.0 & -7.6 & -3.6 \\
\hline 11 & Proximal RCA & RCA & -6.7 & 1.9 & 3.3 & -8.5 & 1.5 & 9.6 & 6.8 \\
\hline 12 & $\begin{array}{l}\text { Proximal RCA, mid } \\
\text { RCA }\end{array}$ & RCA & 1.9 & 2.0 & -1.8 & 33.1 & -6.2 & 11.7 & -18.0 \\
\hline 13 & $\begin{array}{l}\text { Mid RCA (subtotal } \\
\text { occ), proximal Cx (occ) }\end{array}$ & RCA & -0.5 & 1.9 & 0.7 & 15.0 & 29.8 & -16.0 & 6.6 \\
\hline 14 & Proximal RCA & RCA & -9.8 & 2.7 & 1.8 & -13.9 & -17.3 & -10.7 & -4.8 \\
\hline 15 & Mid RCA & RCA & -8.0 & 8.2 & 3.8 & 19.9 & -22.5 & -13.0 & -19.8 \\
\hline 16 & $\begin{array}{l}\text { Mid RCA (subtotal } \\
\text { occ), distal Cx }\end{array}$ & RCA & -1.7 & 1.1 & 1.2 & 0.7 & -30.0 & 1.2 & -29.2 \\
\hline 17 & Mid RCA & RCA & -13.3 & 4.6 & 8.0 & -22.2 & -61.2 & -32.0 & -35.2 \\
\hline 18 & Mid RCA (occ) & RCA & 0.0 & 0.2 & 2.7 & -16.4 & -15.8 & -2.8 & 1.9 \\
\hline \multirow[t]{2}{*}{19} & Proximal RCA & RCA & -5.6 & 4.2 & 0.8 & 29.8 & -6.8 & 60.8 & 29.7 \\
\hline & Mid OM1 & & 3.0 & -0.5 & -0.2 & -2.5 & 36.7 & 2.9 & -5.1 \\
\hline
\end{tabular}

Coronary disease: all potentially significant lesions (minimum cross section $>50 \%$ main vessel) in the preangioplasty coronary arteriogram.

$\mathrm{Cx}$, circumflex coronary artery; D1, 1st diagonal branch of left anterior descending coronary artery; $\mathrm{dP} / \mathrm{dtmax}$, maximum value of 1 st derivative of pressure with respect to time; LAD, left anterior descending coronary artery; maxP, maximum pressure; maxV, minV, maximum and minimum ventricular volumes; occ, occlusion; OM1, 1st obtuse marginal branch of circumflex coronary artery; RCA, right coronary artery; sv, stroke volume; sw, stroke work.

that may occur during the acute and chronic presentations of ischaemic heart disease.

\section{Methods}

SUBJECTS

Patients were studied at the time of therapeutic coronary angioplasty (PTCA). The results of previous diagnostic angiography were known, and patients with appropriate coronary anatomy were selected prospectively. Subjects had significant lesions in either the right coronary artery proximal to the major right ventricular branch or in the left anterior descending coronary artery or its major diagonal branch (table 1). Subjects had single vessel disease, or coexistent disease in non-dominant circumflex coronary arteries. All patients had chronic stable angina, and none had coexistent valvar heart disease or clinically decompensated left ventricular failure. Nineteen subjects were studied. Their mean (SD) age was 58 (8.6) years. Seventeen were male and two female.

Previous angiography had shown angiographically normal left ventricular function in 18 of the 19 subjects, while one, who was undergoing PTCA to the left anterior descending artery, had an anterior hypokinetic segment with well preserved function overall. In no patient was there clinical evidence of infarction in the period between diagnostic angiography and PTCA.

Routine drug treatment included a $\beta$ blocker in 13, a calcium channel blocker in 13, an oral nitrate in 13, enalapril in two (both with left anterior descending coronary artery stenosis), frusemide in two (both with left anterior descending coronary artery stenosis), and aspirin in all 19.
In accordance with the Helsinki agreement all subjects gave informed consent to a protocol approved by the local ethics committee.

PROTOCOL

Patients undergoing PTCA were fasted on the day of the procedure, omitting regular medication. There was therefore a minimum period of 12 hours since the last dose of any drug, and more than 24 hours for once daily drugs. Patients received $10 \mathrm{mg}$ temazepam one hour before PTCA, or no premedication. A $7 \mathrm{~F}$ pigtail diagnostic catheter (Cordis, UK) and a $7 \mathrm{~F}$ pigtail conductance catheter (Webster, USA) (eight electrodes over 60,70 , or $80 \mathrm{~mm}$ according to estimated right ventricular size) were introduced into the right ventricle from the right femoral vein under fluoroscopy. We have previously found that a conductance catheter positioned with the pigtail in the right ventricular apex, describing a curve following the approximate long axis of the right ventricle to the right heart border of the cardiac silhouette, gives an optimal volume signal with respect to signal to noise ratio, phase relation, and stability. ${ }^{12}$ This position was achieved in all cases. A $2.5 \mathrm{~F}$ catheter mounted micromanometer (Millar Instruments, Houston, Texas, USA) was introduced into the diagnostic pigtail catheter to within $50 \mathrm{~mm}$ of the end to give a stable right ventricular pressure trace. Pressure and volume signals were optimised and calibrated, and recorded and displayed continuously as functions of each other to give pressure-volume cycles. After ensuring the correct phase relations of all segmental volumes, only total volume (sum of segmental volumes) was recorded and analysed.

PTCA of the target lesion was performed using femoral guide catheters, monorail 

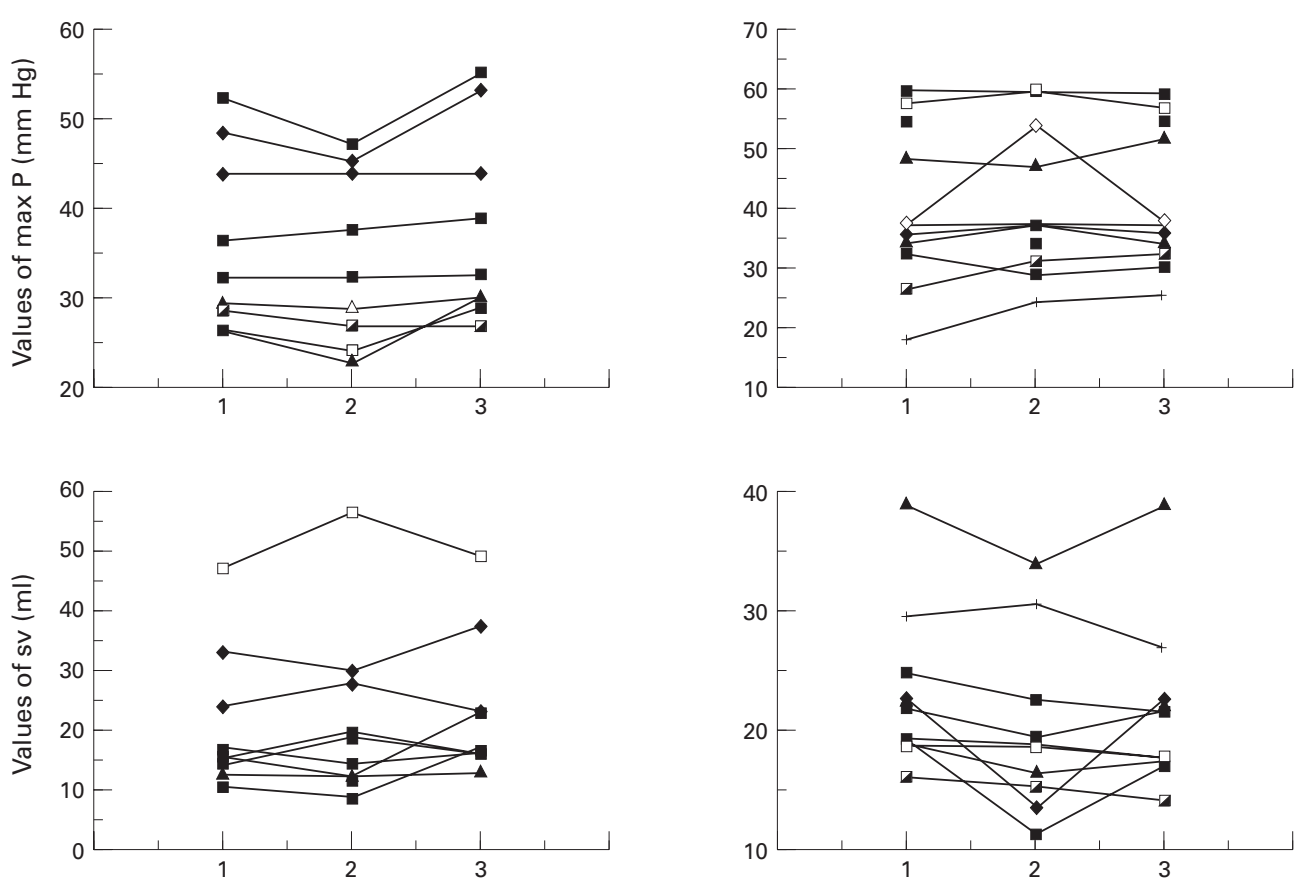

Point of data collection

Figure 1 Values of maximum right ventricular pressure (maxP) and conductance derived stroke volume (sv) in subjects undergoing coronary angioplasty. Values are displayed before balloon inflation (1), after 60 seconds of inflation (2), and 60 seconds after balloon deflation (3). Data from right coronary artery inflations are shown on the left and from left anterior descending coronary artery inflations on the right.

angioplasty catheters, and intracoronary stents as clinically directed. All procedures involving PTCA to the left anterior descending coronary artery resulted in an angiographically successful dilatation of a significant lesion (narrowest luminal cross section $<70 \%$ of the main vessel), without complication. In one patient undergoing PTCA in the right coronary artery, the previous stenosis had become occluded, and attempted disobliteration was unsuccessful. In two other cases the final result was angiographically poor because of intimal dissection. One of these patients underwent emergency coronary artery bypass grafting. At the end of the procedure all catheters were removed, and routine care of the vascular access sites and patient condition was as directed by clinical protocols. In no patient was there a complication associated with the conductance catheterisation.

DATA ACQUISITION AND ANALYSIS

The conductance derived volume signal was generated and processed by a Sigma 5DF single field signal conditioning and processing unit (Leycom, The Netherlands), using a value of blood resistivity determined immediately before each data acquisition. It was transferred directly into custom software in a dedicated microcomputer, through a 12 bit 16 channel analogue to digital (A/D) converter (Data Translation, Wokingham, Berkshire, UK. Segmental volumes were checked for phase relation to simultaneous pressure and intracavity ECG signals, and centred in the $\mathrm{A} / \mathrm{D}$ ranges. We have found previously ${ }^{12}$ that right ventricular conductance derived volume signals have calibration constants (parallel conductance and gain constant relating conductance and thermodilution de- rived stroke volumes) that are robust during physiological volume change; correction for these constants, however, introduces significant error into estimated values of absolute volume, and since no comparison of absolute values was performed between subjects, uncorrected conductance volumes were displayed and recorded. The micromanometer was zeroed to atmospheric pressure at mid-chest level, and calibrated against a standard signal from an amplifier. The pressure signal was imported directly into the same software as the volume signal and displayed and recorded in real time.

Simultaneous pressure and volume data were recorded immediately before the first balloon inflation, after 60 seconds of inflation and 60 seconds after deflation in the coronary artery. Each inflation lasted 60 seconds. In two patients a second lesion in the circumflex coronary artery was treated during the same procedure, and data were acquired during the initial inflation at the second site. Data were acquired over 10 seconds, with respiration suspended after exhaling. Four or five consecutive sinus beats were selected for analysis, and mean values of pressure and volume used in subsequent calculations. In 17 patients data were recorded after completion of the angioplasty procedure.

From pressure-time, volume-time, and pressure-volume relation values of the following were derived or calculated:

$\operatorname{maxV}, \operatorname{minV}$ : maximum and minimum volumes

maxP, minP: maximum and minimum pressures

stroke volume: $\operatorname{maxV}-\operatorname{minV}$

stroke work: area enclosed by the 
Table 2 Pooled data from inflations in the right (RCA) and left anterior descending (LAD) coronary arteries. Mean percentage changes in indices of pump function between onset of balloon inflation and after 60 seconds of coronary artery occlusion are shown

\begin{tabular}{|c|c|c|c|c|}
\hline & \multicolumn{2}{|c|}{ RCA inflation $(n=9)$} & \multicolumn{2}{|c|}{ LAD inflation $(n=10)$} \\
\hline & $\%$ Change & $p$ & $\%$ Change & $p$ \\
\hline Maximum pressure & $-4.8(5.1)$ & 0.02 & $9.9(16.3)$ & 0.04 \\
\hline Maximum volume & $3.0(2.4)$ & 0.004 & $0.9(2.6)$ & 0.16 \\
\hline Minimum volume & $2.3(2.7)$ & 0.01 & $3.7(5.0)$ & 0.02 \\
\hline Stroke volume & $4.2(20.8)$ & 0.16 & $-13.3(15.8)$ & 0.008 \\
\hline Stroke work & $-13.5(16.5)$ & 0.04 & $-10.8(18.7)$ & 0.05 \\
\hline $\mathrm{dP} / \mathrm{dtmax}$ & $0.9(26.2)$ & 0.30 & $12.0(19.2)$ & 0.09 \\
\hline Cycle efficiency & $-14.2(24.6)$ & 0.04 & $-20.9(35.7)$ & 0.02 \\
\hline
\end{tabular}

Vales are mean (SD).

$\mathrm{dP} / \mathrm{dtmax}$, maximum value of 1 st derivative of pressure with respect to time.

$\mathrm{dP} / \mathrm{dtmax}:$

pressure-volume cycle maximum value of the first derivative of pressure against time

cycle efficiency: stroke work $/[(\operatorname{maxV}-\operatorname{minV})$ $\times(\operatorname{maxP}-\min \mathrm{P})]$

There are theoretical and practical reservations about the derivation of exponential or linear relations to describe diastolic pressurevolume relations from cycles at steady state. In contrast to conductance catheter derived pressure-volume cycles described in the left ventricle, the lower right hand corner (end diastole) of cycles from the right ventricle often shows signal noise, probably associated with local turbulence during tricuspid valve closure. Analysis of steady state cycles in previous studies in the left ventricle has suggested changes in the diastolic pressure-volume relations that are misleading when compared to those formally derived by transient vena caval occlusion. ${ }^{13}{ }^{14}$ For these reasons such relations are not reported in this study. However, an obvious distinction between a change in the trajectory or a parallel vertical shift of the diastolic portion of the pressure-volume cycle could be made and is reported.

Since absolute values of volume were not calculated, as discussed above, percentage changes in individual indices during coronary artery occlusion were calculated to allow comparisons between subjects and groups of subjects. Since absolute volumes were not used, ejection fractions were not calculated or compared.

Data from inflations in the right and left anterior descending coronary arteries were analysed as separate groups. Within each group values of the indices described above were compared before balloon inflation and after 60 seconds of inflation.

\section{STATISTICS}

Values of pressure and volume and derived indices were compared using Wilcoxon's sign rank test. A value of $\mathrm{P}<0.05$ was considered significant. Values are quoted as mean (SD).

\section{Results}

CHANGES IN INDICES ASSOCIATED WITH

CORONARY ARTERY OCCLUSION

Table 1 shows the percentage change in all indices that occurred during balloon inflation in all patients. Conductance derived volumes were not corrected for parallel conductance or gain constant, and absolute values of volume derived indices were therefore not compared. Figure 1 shows values of $\operatorname{maxP}$ and stroke volume before inflation, after 60 seconds of inflation, and 60 seconds after deflation, showing that in the majority of cases the changes seen during balloon occlusion had recovered within one minute. This pattern was common to all indices studied. There was no significant or consistent change in heart rate during inflations.

Table 2 compares pooled data from occlusions in the right and left anterior descending coronary arteries.

PATTERNS OF CHANGE ASSOCIATED WITH CORONARY ARTERY OCCLUSION

During right coronary artery occlusion these changes corresponded to a characteristic shift in the pressure-volume cycle predominantly rightward but not upward (fig 2). This pattern of shift was seen during all inflations in a previously patent right coronary artery. During left anterior descending coronary artery occlusion these changes corresponded to a dominant pattern of shift of the pressure-volume cycle upwards but not rightward, with an increase in minV associated with a reduction in stroke volume (fig 2). This pattern was seen during most, but not all, inflations (seven of 10).

Inflations in two separate vessels

In two patients separate inflations were recorded from two different coronary arteries. In one (patient 10) PTCA to the proximal left anterior descending artery was followed by PTCA to the first marginal branch of circumflex artery. In the second (patient 19), PTCA to a dominant right coronary artery was followed by PTCA to the first marginal branch of the circumflex artery. Data from the first patient showed the characteristic pattern of shift associated with left anterior descending artery inflation, and no change during the subsequent circumflex artery inflation. Data from the second showed the characteristic pattern of shift during the right coronary artery inflation, and a different pattern, usually associated with inflations in the left coronary artery, during the inflation in the circumflex artery.

Inflations associated with no change

During balloon inflation in a previously occluded artery (patient 16) there was no apparent shift in the pressure-volume cycles, as expected. During occlusion of a marginal branch of a non-dominant circumflex artery in a patient who had already undergone PTCA to the left anterior descending artery (patient 10), in the same procedure there was also no apparent shift in the pressure-volume cycles (see above). All other inflations were associated with a shift in the pressure-volume cycles.

Changes at the end of the procedure

Data were available at steady state before and at the end of the whole PTCA procedure in 17 cases. In these there was a significant increase in stroke work (mean increase 59.1(95.5)\%, $\mathrm{p}=0.001$ ), and $\mathrm{dP} / \mathrm{dtmax}$ (mean increase $24.1(35.8) \%, p=0.01)$ associated with an 
A

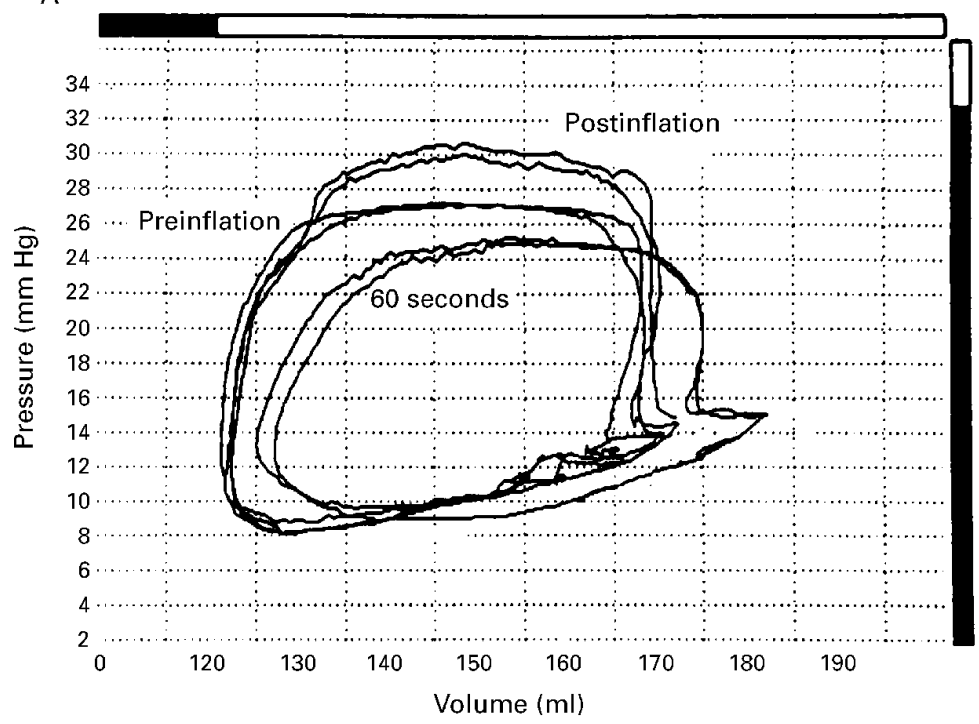

B

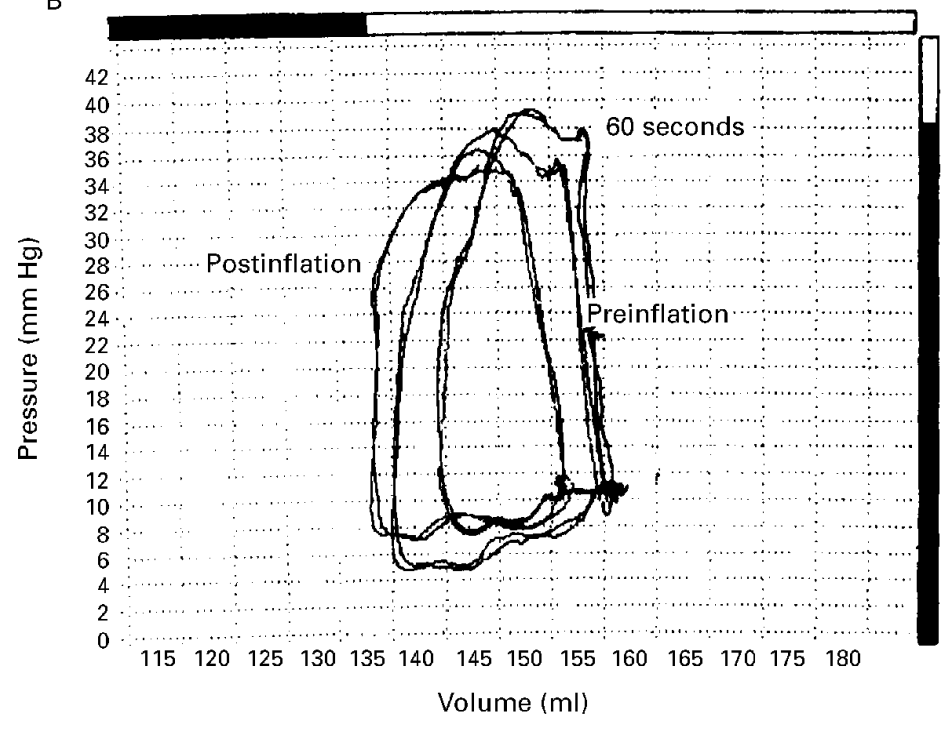

Figure 2 Right ventricular pressure-volume cycles from patients undergoing coronary angioplasty to lesions in the right coronary artery (above) and left anterior descending coronary artery (below). Two characteristic patterns of shift are contrasted with predominant rightward or vertical displacement. Vertical displacement is associated with a reduction in stroke volume, which in this subject results from a predominant reduction in maximum ventricular volume, while stroke volume during right ward displacement is maintained.

increase in maxP (mean increase $16.7(19.8) \%$, $\mathrm{p}=0.01)$. These were interpreted as the non-specific consequences of general neurohumoral activation. There was no significant change in volumes or cycle efficiencies.

Changes in diastolic pressure-volume relations Constants describing a theoretical diastolic pressure-volume relation were not derived. However, changes in the diastolic portions of the pressure-volume cycles were often apparent. During 10 of 19 inflations there was a clear dominant vertical shift in the whole diastolic pressure-volume relation with little apparent change in its trajectory. This was most often observed in association with a dominant upward shift in the pressure-volume cycle (eight of nine inflations), and rarely in association with a rightward shift (two of 10 inflations). This is illustrated in fig 3. This phenomenon was therefore a feature of balloon inflations in the left anterior descending coronary artery. In all other cases the diastolic portion of the pressure-volume cycle appeared to follow a continuous relation during inflations.

There were not sufficient data for useful subgroup analysis, but the presence of obvious collaterals (identified on the angiogram immediately before angioplasty: patients $12,13,16$ ), or abnormal resting left ventricular function (patient 2), were associated with less marked changes in pressure and volume derived indices.

\section{Discussion}

This study shows that in subjects with ischaemic heart disease, coronary artery occlusion was associated with the rapid development of changes in right ventricular pressure-volume relations. One of the advantages of simultaneous pressure and volume measurement is that ventricular stroke work can be calculated and related to changes in pressure and volume which usually reflect changes in ventricular loading. In this way changes in contractile function may be distinguished from changes in the preload and afterload of the ventricle. The data presented here show that there was a decline in stroke work associated with occlusion of both right and left anterior descending coronary arteries. The changes in pressure and volume, however, were not uniform, but suggest two distinct patterns: (1) during occlusion of a dominant right coronary artery there was an increase in maximum and minimum ventricular volume associated with no rise or a fall in maximum pressure; (2) during occlusion of left anterior descending coronary artery, there was usually a rise in maximum pressure and a decline in stroke volume. These different patterns of change in right ventricular pump function support the intuitive view that coronary artery occlusion may modify right ventricular contractile function and loading in different ways, depending on the site of the resulting ischaemia.

\section{PREVIOUS STUDIES}

Adverse haemodynamic changes associated with right ventricular infarction are well recognised clinically, ${ }^{15}$ with important effects on short term prognosis independent of left ventricular function. ${ }^{5}$ However, detailed analysis of right ventricular function during coronary artery occlusion has not been done in human subjects. Several studies have reported that both right coronary and left anterior descending coronary artery occlusion are associated with a decline in right ventricular ejection fraction. ${ }^{16-19}$ Since right ventricular ejection fraction is sensitive both to impaired right ventricular contractile function and to a rapid increase in pulmonary artery pressure, a fall in ejection fraction does not distinguish direct and indirect effects of cardiac ischaemia. However, a rise in pulmonary artery pressure, ${ }^{16}{ }^{19}$ and thus right ventricular systolic pressure, associated with an increase in low 


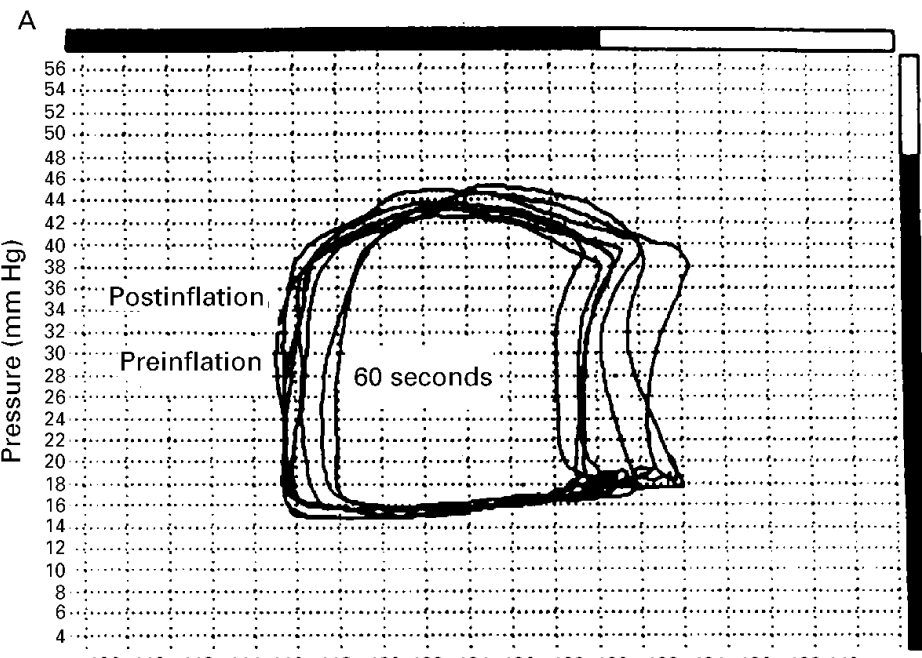

$\begin{array}{lllllllllllllllll}106 & 110 & 112 & 114 & 116 & 118 & 120 & 122 & 124 & 126 & 128 & 130 & 132 & 134 & 136 & 138 & 140\end{array}$

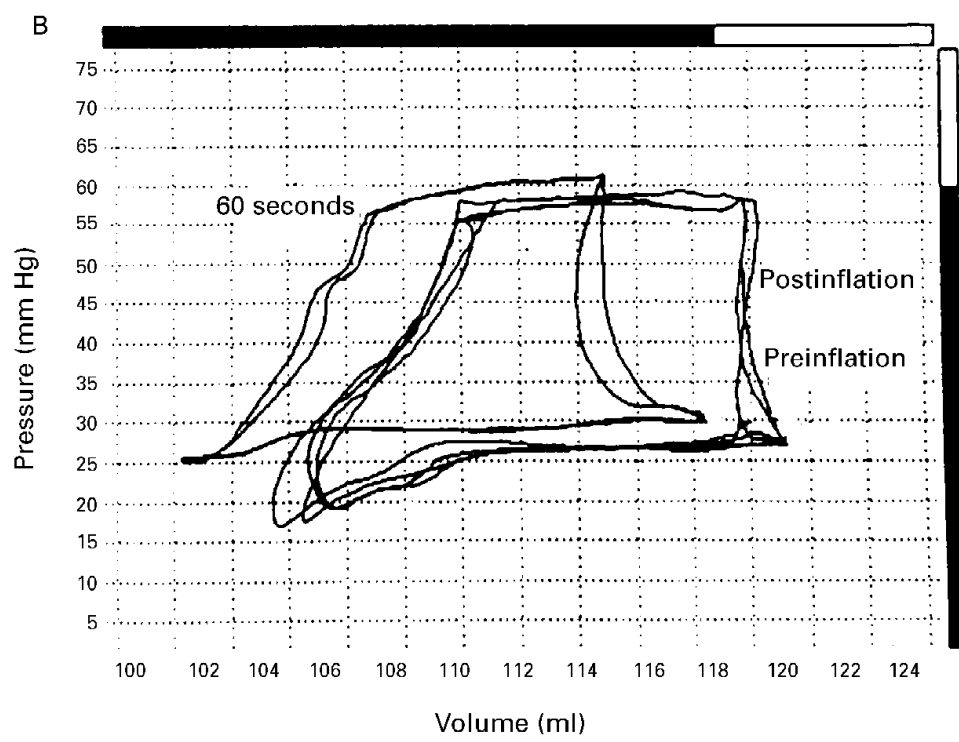

Figure 3 Right ventricular pressure-volume cycles from patients undergoing coronary angioplasty to lesions in the right coronary artery (above) and left anterior descending coronary artery (below). Two characteristic patterns of change in the diastolic

pressure-volume relations are contrasted. The cycles in the top figure follow a continuous relation, and those in the bottom figure are displaced vertically during coronary artery occlusion. previous findings, ${ }^{16}{ }^{17}$ and biologically plausible, assuming that the effects of left anterior descending coronary artery occlusion on left ventricular diastolic performance are more significant than right coronary artery occlusion, and that these effects lead to changes in the pulmonary circulation that result in an increase in impedance. ${ }^{20} \mathrm{~A}$ more unexpected finding was that while the maximum ventricular volume increased during right coronary artery occlusion, this was less consistently observed during occlusion of the left anterior descending coronary artery, so that for pooled data the change was not significant (table 2). During occlusion of either vessel the minimum (that is, end systolic) ventricular volume increased, but during right coronary artery occlusion the increase in maximum (that is, diastolic) ventricular volume had the effect that stroke volume did not decline significantly. In contrast, failure to increase the diastolic volume during left anterior descending coronary artery occlusion resulted in a significant fall in stroke volume. Maximum ventricular volume is functionally the best index of ventricular preload. In simple terms, therefore, during right coronary artery occlusion there was an increase in preload and no rise in afterload, while during left anterior descending coronary artery occlusion there was a rise in afterload but no consistent rise in preload.

The Frank-Starling mechanism operates to maintain pump function in the face of an increased ventricular afterload or reduced ventricular contractile function by an increase in ventricular diastolic volume, as seen during right coronary artery occlusion. Its failure, and the resulting decline in stroke volume during occlusion of the left anterior descending coronary artery, is unexpected. Right ventricular diastolic dilatation may be mechanically constrained by the pericardium, or by movement of the interventricular septum into the right ventricular cavity during left ventricular dilatation; the latter effect may explain failure of right ventricular dilatation during left anterior descending coronary artery occlusion, since acute ischaemic left ventricular dilatation would be anticipated. The patterns of change observed in the diastolic right ventricular pressure-volume relations (fig 3) support this explanation: an increase in the gradient of the diastolic pressure-volume relation implies an increase in ventricular stiffness, but a parallel upward shift of the relation is a feature of mechanical constraint of the ventricle, for example during acute volume loading of the right ventricle, ${ }^{21}$ or during acute ischaemia of the left ventricle. ${ }^{15} \mathrm{~A}$ predominantly parallel shift was a common observation during occlusion of the left anterior descending coronary artery.

\section{RIGHT VENTRICULAR CONTRACTILE FUNCTION}

In the left and right ventricles a positive linear relation between stroke work and end diastolic volume is recognised. ${ }^{22}{ }^{23}$ The gradient of this relation is insensitive to changes in ventricular load but sensitive to changes in contractile state. During inflations in the right coronary coronary artery implies an increase in ventricular afterload. This difference is consistent with 
artery and left anterior descending coronary artery, the usual effect was a fall in stroke work, and in the absence of a reduction in maximum volume, this effect implies a decline in contractile function, that is, a reduction in the gradient of the stroke work-end diastolic volume relation. This is in keeping with observations in animal models of the effects of coronary artery ligation on right ventricular contractile function. The right coronary artery in the canine heart supplies only the right ventricle, and its ligation causes a fall in right ventricular $\mathrm{dP} / \mathrm{dtmax},{ }^{24}$ the end systolic pressure-volume relation, and the stroke work-end diastolic volume relation. ${ }^{25}$ In pigs, left anterior descending coronary artery ligation results in similar reductions in gradients of the right ventricular end systolic pressure-volume relation ${ }^{26}$ and the stroke work-end diastolic relation. ${ }^{27}$ In the data presented here the changes in stroke work and the implied decline in contractile function suggest a direct effect of ischaemia, presumably as a result of ischaemia in the septum during left anterior descending coronary artery occlusion and in the right ventricular free wall during right coronary artery occlusion.

RIGHT VENTRICULAR dP/dtmax CYCLE EFFICIENCY $\mathrm{dP} / \mathrm{dtmax}$ is often interpreted as an index of contractile function in the left and right ventricles. In the subjects in this study it did not change significantly during coronary artery occlusion, and there was a trend towards an increase in subjects in whom maxP increased. Its sensitivity to changes in afterload and insensitivity to change in contractile state thus limit its usefulness in this context. Cycle efficiency is an index of the extent to which the ventricular function approaches that of an ideal square wave pump. The low cycle efficiency of the normal right ventricle is a reflection of the low impedance and high capacitance of the pulmonary circulation, and is increased in the presence of an increased ventricular afterload. ${ }^{28}$ The fall in cycle efficiency that occurred during right and left anterior descending coronary artery occlusions is not easily explained, and argues against interpretation of this index simply as reflecting the coupling between the right ventricle and pulmonary circulation.

\section{CRITICISM OF METHODS}

One marked feature of continuous right ventricular pressure and volume recording is the sensitivity of all measurements to respiratory movement and other physical activity. This variability is not apparent in most reported studies of right ventricular function, since they are usually performed in anaesthetised and ventilated animals, as described in recent reports of the use of conductance catheters in the pig right ventricle. ${ }^{10}$ Studies of human right ventricular pressure-volume relations have not previously used techniques that would be sensitive to such variation (for example, angiography, radionuclide ventriculography). Against this background, two observations support the validity of the changes reported here in association with coronary artery occlusion. Firstly, in one patient in whom the target lesion for PTCA had progressed to subtotal occlusion, no changes in the right ventricular pressure-volume relations were observed while the lesion was crossed with a catheter and the balloon inflated. Secondly, the changes recorded during inflations in previously patent arteries formed clearly recognisable patterns, most clearly distinguished in patient 19 , in whom inflations in right coronary and circumflex coronary arteries had different consequences during the same procedure.

The use of conductance catheters in adult human subjects is technically demanding, and while there are many reports describing the validation and use of the technique in small animal experiments in the left, and more recently right, ventricle, reports in adult human subjects are scarce and describe their use in the left ventricle, where the cavity geometry is less complex. Our experience of the technique in the conscious adult human right ventricle suggests that it is often difficult but that it is important to be rigorous in differentiating real change from artefact. During coronary artery occlusion three potential sources of artefact may contribute to apparent volume change.

Firstly, since conductance derived right ventricular volumes are sensitive to respiration it is important that all volumes are measured at the same respiratory phase, for which patient compliance is important in the unanaesthetised subject.

Secondly, changes in the cavity size and geometry during ischaemia may alter the calibration constants relating real to conductance volumes, distorting the relation between volume change and changes in the conductance signal. We have previously investigated the stability of parallel conductance (contribution of extracavity conductance to the overall conductance volume) and the gain constant relating conductance and thermodilution derived techniques during volume expansion, ${ }^{12}$ and found no significant or systematic change. The configurational changes that occur in the right ventricular cavity during ischaemia are probably more complex than those during simple volume expansion, but are of the same magnitude, and we do not anticipate a significant systematic change in parallel conductance or gain constant during the changes described in this data.

Thirdly, parallel conductance is determined in part by the patterns of anisotropic conductivity in the ventricular myocardium. These patterns are likely to be sensitive to changes in perfusion, and thus coronary artery occlusion may cause a change in parallel conductance and a volume independent change in conductance volume. If this effect were significant it is unlikely that consistent patterns of volume change with respect to pressure change would result, and such patterns argue strongly that the changes observed in these data are indices of real physiological change.

There are two assumptions in the interpretation of the changes of right ventricular pressure-volume data as the effects of ischaemia. Firstly, it is assumed that coronary 
artery occlusion results in functional ischaemia with effects within the first minute, and secondly, it is assumed that the site of occlusion predicts the location of functional ischaemia. There are several reports describing the effects of coronary artery occlusion during PTCA on indices of left ventricular function, ${ }^{14-31}$ and changes in diastolic and systolic indices within one minute are well recognised. Since the majority of subjects described here were thought to have functionally single vessel coronary artery disease, it is likely that interpretation of changes in association with occlusion in a particular artery as representing the effects of ischaemia in an associated area of myocardium are valid. The distinct patterns observed with particular inflation sites support this view.

Analysis of the changes in the ventricular pressure-volume cycle that are observed with rapid changes in loading (for example, inferior vena caval occlusion) extends the information that can be gained from such studies. Without such manoeuvres, shifts in the pressurevolume cycles as reported here are interpreted by assuming the existence of relations (for example, preload recruitable stroke work) without formally proving their existence. In ex vivo $^{32}$ and in vivo ${ }^{10}$ animal preparations, these relations are demonstrable in the right ventricle, supporting their assumption in interpretation of the changes seen in these subjects.

\section{CONCLUSIONS}

Despite the technical and theoretical reservations discussed, the changes in the right ventricular pressure-volume relations observed in these subjects clearly reflect changes in the pump function of the right ventricle during coronary artery occlusion. These changes can be interpreted within a framework that distinguishes active and passive properties of the ventricle and its loading conditions. Occlusion of the right coronary artery and of the left anterior descending coronary artery lead to a decline in the stroke work of the right ventricle. However, the effects of this decline on pump function reflect changes in the loading conditions of the ventricle. Pump failure - a reduction in stroke volume-is a consistent feature when there is a rise in peak ventricular pressure and a failure to augment the diastolic volume.

Andrew Bishop was a Junior Fellow of the British Heart FounAation. Much of the equipment described here was provided by the Scott Rhodes Research Fund. The software used in data analysis was provided by Richard Szwarc.

1 Fontan F, Baudet E. Surgical repair of tricuspid atresia. Thorax 1971;26:240-8.

2 Sade R, Castaneda A. The dispensable right ventricle. Surgery 1975;77:624-31.

3 Baker B, Wilen M, Boyd C, Dinh H, Franciosa J. Relation of right ventricular ejection fraction to exercise capacity in chronic left ventricular failure. Am f Cardiol 1984;54:596-

4 Polak J, Holman L, Wynne J, Colucci W. Right ventricular ejection fraction: an indicator of increased mortality in patients with congestive heart failure associated with 5 Zoronary artery disease. F Am Coll Cardiol 1983;2:217-24 Olschewski, Kasper W, Kauder E, Schontaler M, Geibel a, independent predictor of prognosis after acute inferio myocardial infarction. N Engl f Med 1993;328:981-8.

6 Weber K, Janicki J, Shroff S, Fishman A. Contractile mechanics and interaction of the right and left ventricles. Am f Cardiol 1981;47:686-95.
7 Brown K, Okada R, Boucher C, Strauss W, Pohost G. Right ventricular ejection fraction response to exercise in patients with coronary artery disease: influence of both right coronary artery disease, and exercise induced changes in right ventricular afterload. F Am Coll Cardiol 1984:3:89590 .

8 Berger H, Johnstone D, Sands J, Gottschalk A, Zaret B. Response of right ventricular ejection fraction to upright bicycle exercise in coronary artery disease. Circulation 1979;60:1292-9.

9 White P, Bishop A, Conroy B, Oldershaw P, Redington A. The determination of volume of right ventricular casts using a conductance catheter. Eur Heart F 1995;16:1425-9.

10 Dickstein M, Yano O, Spotnitz H, Burkhoff D. Assessment of right ventricular contractile state with the conductance catheter technique in the pig. Cardiovasc Res 1995;29:8206.

11 Stamato T, Szwarc R, Benson L. Measurement of right ventricular volume by conductance catheter in closed-chest pigs. Am F Physiol 1995;269:H869-76.

12 Bishop A, White P, Oldershaw P, Chaturvedi R, Redington A. The use of conductance catheters to assess human right entricular volumes: in vivo and in vitro validation [abstract]. Eur Heart f 1995;16:310(P1754).

13 Applegate R. Load dependence of left ventricular diastolic pressure-volume relations during short term coronary artery occlusion. Circulation 1991;83:661-73.

14 Kass D, Midei M, Brinker J, Maughan L. Influence of coronary occlusion during PTCA on end-systolic and end diastolic pressure-volume relations in humans. Circulation 1990;81:1447-60.

15 Cohn J, Guiha N, Broder M, Constantinos L. Right ventricular infarction. Am f Cardiol 1974;33:209-14.

16 Danchin N, Julliere Y, Schrijen F, Cherrier F. Differential effects on right ventricular function of transient right, left anterior descending, and left circumflex coronary occlusions during percutaneous transluminal coronary angioplasty. $₹$ Am Coll Cardiol 1991;18:437-42.

17 Danchin N, Julliere Y, Schrijen F, Cherrier F. Right ventricular function during transient myocardial ischaemia induced by percutaneous transluminal angioplasty of the proximal right coronary artery. Am f Cardiol 1989;63:98991

18 Mauser M, Karsch K, Seipel L. Effect of right coronary occlusion during percutaneous transluminal coronary angioplasty on right ventricular performance. Am f Cardiol 1988;61:648-50.

19 Burger W, Hansen C, Allroggen H, Kober G. Effects of exercise or temporary coronary occlusion during angioplasty on right ventricular function with consideration of left anterior descending coronary artery related myocardial ischaemia. Cardiology 1993;83:345-57.

20 Kussmaul W, Wieland J, Laskey W. Pressure-flow relations in the pulmonary artery during myocardial ischaemia: implications for right ventricular function in coronary disimplications for right ventricular functio

21 Dell'Italia L, Walsh R. Right ventricular diastolic pressurevolume relations and regional dimensions during acute alterations in loading conditions. Circulation 1988;77: 1276-82.

22 Glower D, Spratt J, Snow N, Kabas J, Davis J, Olsen C, et al. Linearity of the Frank-Starling relationship in the intact heart: the concept of preload recruitable stroke work. Circulation 1985;71:994-1009.

23 Karunanithi M, Michniewicz J, Copeland S, Fenely M. Right ventricular preload recruitable stroke work, endsystolic pressure-volume, and $\mathrm{dP} / \mathrm{dtmax}$-end diastolic contractile performance in conscious dogs. Circ Res 1992;70:1169-79.

24 Brooks H, Holland R, Al-Sadir J. Right ventricular performance during ischaemia: an anatomic and hemodynamic ance during ischaemia: an anatomic an

25 Moon M, Castro L, DeAnda A. Effects of left ventricular support on right ventricular mechanics during experimental right ventricular ischaemia [abstract]. Circulation 1994; 90:II-92-101

26 Krams R, Soei L, McFalls E, Prins E, Sassen LM, Verdouw P. End-systolic pressure-length relations of stunned right and left ventricles after inotropic stimulation. Am f Physiol 1993;265:H2099-109.

27 Uppal R, Craig D, Glower D, Smith P. Effects of inotropic agents on post ischaemic right ventricular systolic performance and pulmonary impedance [abstract]. Eur Heart $f$ 1993;14:439(2470).

28 Redington A, Rigby M, Shinebourne E, Hodgson M, Oldershaw $\mathrm{P}$. Changes in the pressure-volume relation of the right ventricle when its loading conditions are changed. $\mathrm{Br}$ Heart f 1990;63:45-9.

29 Sigwart U, Grbic M, Essinger F, Morin D, Sadeghi H. Myocardial function in man during acute coronary balloon occlusion [abstract]. Circulation 1982;66(suppl II):II-86 (344)

30 Serruys P, Wijns W, Van der Brand M, Meij S, Slager C, Schuurbiers J, et al. Left ventricular performance, regional blood flow, wall motion, and lactate metabolism during transluminal angioplasty. Circulation 1984;70:25-36.

31 Bertrand M, Lablanche J, Fourrier J, Traisnel G, Mirsky I. Left ventricular systolic and diastolic function during acute coronary artery balloon occlusion in humans. F Am Coll Cardiol 1988;12:341-7.

32 Maughan L, Shoukas A, Sagawa K, Weisfeldt M. Instantaneous pressure-volume relationship of the canine right ventricle. Circ Res 1979;44:309-15. 